\title{
Symmetry and selection rules for localized surface plasmon resonances in nanostructures
}

\author{
Weihua Zhang (张伟华), ${ }^{*}, \dagger$ Benjamin Gallinet, ${ }^{\ddagger}$ and Olivier J. F. Martin ${ }^{*}, \$$, \\ Nanophotonics and Metrology Laboratory, Swiss Federal Institute of Technology Lausanne (EPFL), 1015 Lausanne, Switzerland
}

(Received 8 April 2010; published 29 June 2010)

\begin{abstract}
We describe a general theoretical framework based on the Bergman spectral representation to study how a nanostructure interacts with an external electromagnetic field. The selection rules for localized surface plasmon resonances (LSPRs) are obtained by implementing the group theory upon the electric vector field. The influence of symmetry breaking on the splitting of degenerated modes and the switching of dark modes by specific illuminations are discussed. These results emphasize the fact that the selection rules for a vector field are different from the case of a scalar field and essentially induced by the geometry of the structure. Finally, this work not only points out that measurements of LSPRs may result in very different results with different external fields, but also provides a strategy to selectively excite specific LSPRs of plasmonic structures.
\end{abstract}

DOI: $10.1103 /$ PhysRevB.81.233407

PACS number(s): 78.67.Bf, 02.20.-a, 73.20.Mf, 78.20.Bh

In the optical regime, a metal nanostructure can interact strongly with photons by virtue of the collective oscillation modes of the free electrons, namely, the localized surface plasmon resonances (LSPRs). ${ }^{1}$ Some of these resonance modes - the bright modes (i.e., radiative modes) — bridge the electromagnetic fields between the near-field and far-field zones of the structure, letting the nanostructure function as an optical antenna. ${ }^{2,3}$ Other resonance modes-the dark modes (i.e., nonradiative modes) — are tightly bound to the surface of the nanostructure and do not couple with light propagating in the far-field zone. These dark modes produce a nanometer-sized resonator which is essential for generating coherent surface plasmons. ${ }^{4}$ Interestingly, the interaction between the different LSPR modes and the photons depends on the symmetry of both the nanostructure and the external field, which can be either the incident or the scattered field. Indeed, recent reports indicate that the optical properties of plasmonic nanostructures can be controlled by changing the symmetry of the structure or that of the external field. ${ }^{5-7}$ Inspired by this idea, in this work we develop a theoretical framework to investigate the influence of the symmetry of the system on the LSPRs using the Bergman spectral representation. This approach allows us to solve the photon-LSPR interaction as an expansion series of eigenmodes. ${ }^{8,9}$ This will allow us to establish selection rules for the interaction of LSPR with different types of external field, namely, plane wave excitations, spatially modulated fields, and electrical dipolar fields.

The Bergman representation was originally developed to determine the effective refractive index of composite materials and later implemented for studying the LSPRs associated with nanostructures. ${ }^{8,9}$ In this work, we use the Bergmann spectral representation combined with the Green's tensor method in order to separate the excitation field from the boundary conditions. ${ }^{10}$ This allows us to handle arbitrary external fields.

Let us first recall briefly the theory and consider an arbitrary shape object with permittivity $\varepsilon$ in vacuum. The scattering problem in the frequency domain can be described with Lippmann-Schwinger equation,

$$
\mathbf{E}(\mathbf{r})=\mathbf{E}_{\text {ext }}(\mathbf{r})+\int_{\text {scatter }} d \mathbf{r}^{\prime} \Delta \varepsilon\left(\mathbf{r}^{\prime}\right) k_{0}^{2} \mathbf{G}_{0}\left(\mathbf{r}, \mathbf{r}^{\prime}\right) \cdot \mathbf{E}\left(\mathbf{r}^{\prime}\right),
$$

where $\mathbf{E}_{e x t}$ is the external field and $\mathbf{G}_{0}$ is the dyadic Green's tensor. ${ }^{11}$ Defining $[\hat{\mathbf{G E}}](\mathbf{r})=\int_{\text {scatter }} d \mathbf{r}^{\prime} \mathbf{G}_{0}\left(\mathbf{r}, \mathbf{r}^{\prime}\right) \cdot \mathbf{E}\left(\mathbf{r}^{\prime}\right) k_{0}^{2}$, Eq. (1) can be rewritten as $\mathbf{E}=\mathbf{E}_{e x t}+\frac{1}{s} \hat{\mathbf{G E}}$, where $s=1 / \Delta \varepsilon$ $=1 /(\varepsilon-1)$. This can be solved as an eigenvalue problem $\hat{\mathbf{G E}}=s \mathbf{E}$. Under the quasistatic approximation, the operator $\hat{\mathbf{G}}$ is real and symmetric. Thus, it has a set of real eigenvalues $\left\{s_{\mathrm{n}}\right\}$ and orthonormal eigenmodes $\left\{\mathbf{E}_{\mathrm{n}}\right\}$. The scattered field can be expressed as the sum of these eigenmodes,

$$
\mathbf{E}=\sum_{n} a_{n} \mathbf{E}_{n}
$$

where $a_{n}=\frac{s}{s-s_{n}}\left\langle\mathbf{E}_{n} \mid \mathbf{E}_{\text {ext }}\right\rangle$ is the interaction strength between the $n$th eigenmode and the external field.

In the following, we shall use this formalism to determine the selection rules of LSPRs for different conditions. Considering that the dimension $l$ of the structure is much smaller than the wavelength (i.e., the quasistatic approximation), the external field in the object can be described with the Taylor expansion around the position $\mathbf{r}_{0}$ of the structure,

$$
\mathbf{E}_{e x t}(\mathbf{r})=\mathbf{E}_{e x t}\left(\mathbf{r}_{0}\right)+\sum_{i} \frac{\partial \mathbf{E}_{e x t}\left(\mathbf{r}_{0}\right)}{\partial r_{i}} r_{i}+\cdots .
$$

In the case of a free-space propagating external field, the diffraction limit implies that the second term of the expansion [Eq. (3)], $\frac{\partial \mathbf{E}_{e x t}\left(\mathbf{r}_{0}\right)}{\partial r_{i}} r_{i}$, has the same order of smallness as $l / \lambda$ compared with the $\mathbf{E}_{\text {ext }}\left(\mathbf{r}_{0}\right)$. We can therefore treat $\mathbf{E}_{\text {ext }}$ as a constant vector over the structure. Hence, the excitation strength of mode $n$ is

$$
\left\langle\mathbf{E}_{n} \mid \mathbf{E}_{\text {ext }}\right\rangle=\mathbf{E}_{\text {ext }} \cdot \int_{\text {scatter }} d \mathbf{r} \mathbf{E}_{n}^{*}(\mathbf{r})=\mathbf{E}_{\text {ext }} \cdot \frac{\mathbf{P}_{n}^{*}}{\alpha},
$$

where $\mathbf{P}_{n}$ is the total dipole moment of the $n$th eigenmode of nanostructure and $\alpha$ is the effective polarizability per unit volume of the nanostructure.

Hence, a mode is bright (i.e., interacts with the external field) when there is one component of $\mathbf{P}_{i}$ - the integral in Eq. 
TABLE I. Character table for the group $C_{4 v}$.

\begin{tabular}{lrrrrr}
\hline \hline & $E$ & $2 C_{4}$ & $C_{2}$ & $2 \sigma_{v}$ & $2 \sigma_{d}$ \\
\hline$A_{1}$ & 1 & 1 & 1 & 1 & 1 \\
$A_{2}$ & 1 & 1 & 1 & -1 & -1 \\
$B_{1}$ & 1 & -1 & 1 & 1 & -1 \\
$B_{2}$ & 1 & -1 & 1 & -1 & 1 \\
$E$ & 2 & 0 & -2 & 0 & 0 \\
\hline \hline
\end{tabular}

(4) — that does not vanish. To reveal the role of symmetry here, let us consider a symmetric nanostructure characterized by a point group $\mathcal{G}$. Defining a symmetry operator $\hat{\mathbf{T}}_{g}$ for each element $\mathrm{g} \in \mathcal{G}$, we have the commutation relation $\hat{\mathbf{G}} \hat{\mathbf{T}}_{g}=\hat{\mathbf{T}}_{g} \hat{\mathbf{G}}$. According to group theory, each invariant eigenspace of $\hat{\mathbf{G}}$ carries an irreducible representation (irrep) $\Gamma$ of $\mathcal{G}$. In the case of a scalar field $f$ (e.g., a wave function in quantum mechanics), the selection rules can be found in textbooks: eigenmode $f_{n}$ cannot be excited unless $f_{n}$ is the base of a unit irrep of $\mathcal{G} .^{12}$

In this work however, we are dealing with a vectorial field $\mathbf{E}$ and the selection rules are more complicated than for a scalar field. Indeed, a bright mode may acquire its intensity from any of the three components of $\mathbf{E}$ and these components do not necessarily transform in the same way. For example, in Cartesian coordinates, if we define a rotation transformation $\mathbf{R}_{g}$ for each symmetry operator $\hat{\mathbf{T}}_{g}, \hat{\mathbf{T}}_{g}$ will act on vector field $\mathbf{E}$ as $\left[\hat{\mathbf{T}}_{g} \mathbf{E}\right](\mathbf{r})=\mathbf{R}_{g} \mathbf{E}\left(\mathbf{R}_{g}^{-1} \mathbf{r}\right)$ instead of $\left[\hat{\mathbf{T}}_{g} f\right](\mathbf{r})$ $=f\left(\mathbf{R}_{g}^{-1} \mathbf{r}\right)$ as would be the case for a scalar field $f .{ }^{13,14}$ Hence, in the cases of a sufficiently high symmetry, the transformation of each component will involve other components since $\mathbf{R}_{g}$ also rotates the vector $\mathbf{E}$, and a component of the eigenvector may not transform as an irrep of $\mathcal{G}$. Even in the case of a one-dimensional irrep, the three components of the vector field may be the bases of different irreps. Therefore, the selection rules become in our case: $\mathbf{E}_{n}$ is a dark mode, unless one of its components contains the unit irrep of $\mathcal{G}$.

Let us take a square flake which bears the $D_{4 h}$ symmetry as an example. The profiles of the possible modes can be generated with the help of the character Table I. ${ }^{14,15}$ These modes are shown in Fig. 1(a); for simplicity, we only consider the modes of $C_{4 v}$ in the $x-y$ plane instead of $D_{4 h}$, since all the modes of $D_{4 h}$ can be generalized from the modes of $C_{4 v}$ using $D_{4 h}=C_{4 v} \otimes C_{s}$. There are three types of modes in Fig. 1(a): first, $A_{1}$ which is the electric dipole mode along the principal axis (i.e., the $z$ axis in this case); second, the two degenerated modes of irrep $E$ which correspond to the electric dipole modes in the $x-y$ plane; third $A_{2}, B_{1}$, and $B_{2}$, which correspond to the magnetic dipole mode and electric quadrupole modes.

We can directly determine whether the modes in Fig. 1(a) are bright or not with the help of the selection rules. Mode $A_{1}$ is bright because the $z$ component of the electric field is a base of the unit irrep $A_{1}$. In the case of the two degenerated modes of irrep $E$, either the $x$ or the $y$ component carries both the unit irrep $A_{1}$ and $B_{1}$; therefore these modes are bright. We would like to emphasize that these two degener-
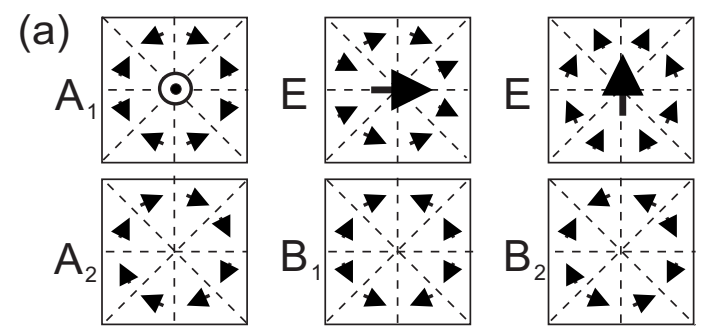

(b)

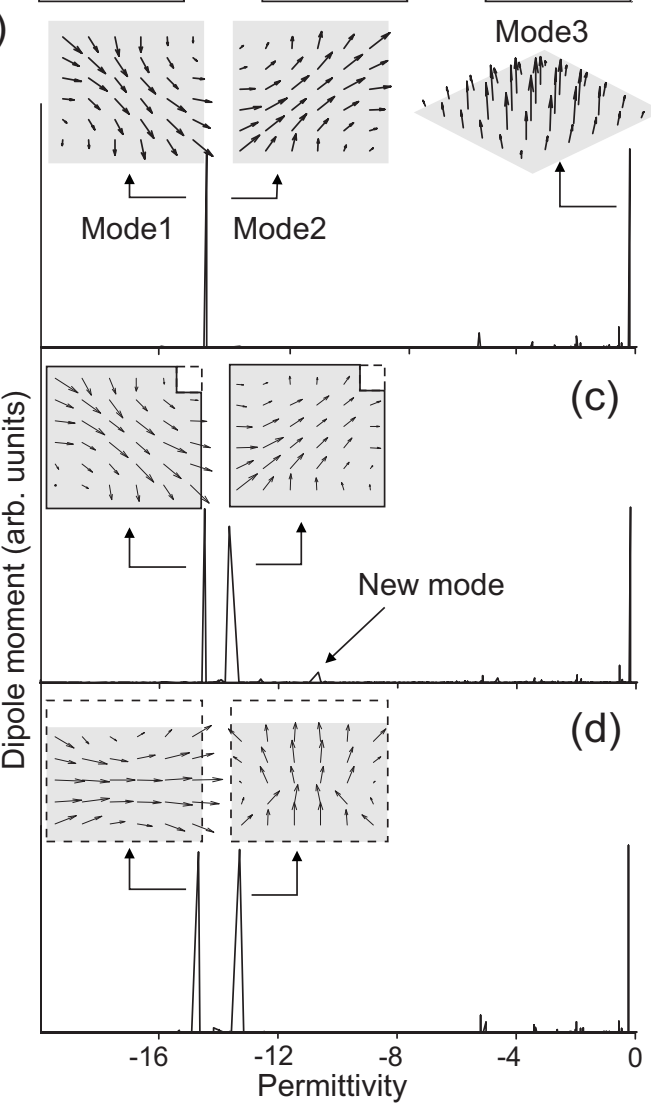

FIG. 1. (a) The possible symmetric modes of $C_{4 v}$ in the $x-y$ plane, the arrows show the electric field vectors. (b) The dipole moments of the LSPR modes as a function of the permittivity (corresponding to the eigenvalues) for a $2.5 \mathrm{~nm}$ thick square flake which dimensions $20 \times 20 \mathrm{~nm}^{2}$. Dipole moment spectra for perturbed structures with (c) a $2 \times 2 \mathrm{~nm}^{2}$ corner removed and (d) a 2 $\mathrm{nm}$ shorter side in $y$ direction. The electric field distributions of the dominant modes appearing in the spectra (b)-(d) are shown in the corresponding insets; the direction and intensity of the electric fields are denoted by the orientation and length of the arrows.

ated $E$ modes would be dark in the case of a scalar field (e.g., in the case of Schrödinger's equation), and they are bright here because the electric field is vectorial. The modes $A_{2}, B_{1}$ and $B_{2}$ on the other hand, do not have any components which carry a representation containing the unit irrep $A_{1}$ and are therefore dark.

We numerically solved the eigenmodes of this square flake by discretizing it into small cubes. The amplitude of the dipole moment $\mathbf{P}_{n}$ associated with the $n$th eigenmode was then calculated and plotted as the function of $\varepsilon_{n}$ (i.e., $1 / s_{n}$ +1 ), Fig. 1(b). Three dominant modes are clearly visible in this figure: the three dipolar modes corresponding to the three different spatial dimensions. Comparing the field dis- 
tributions of these three dominant modes with the modes depicted in Fig. 1(a), we observe that they correspond to the $A_{1}$ mode and doubly degenerated modes of $E$, respectively. Although the two degenerated modes of $E$ in Fig. 1(b) appear different from the sketch in Fig. 1(a), they can be expressed as a linear combination of the two degenerated modes.

In quantum mechanics, it is well known that degenerated modes split when the symmetry of the system is broken and the profiles of the new modes depend on the symmetry of the new structure. To demonstrate this effect, we lower the symmetry of the square flake structure in two different ways: first by removing a corner and second by shortening one side; the symmetry axes then become the diagonal through the broken corner and the new middle lines along the $x$ and $y$ axes. Hence, new modes of the structure are oriented along or perpendicular to the new symmetry axes, as shown in Figs. 1(c) and 1(d).

This mode splitting can also be explained by the perturbation theory. In Fig. 1(c), because the volume of the removed corner is much smaller than the total volume of the original square flake, the new system can be described as $\mathbf{E}=\mathbf{E}_{\text {ext }}+\frac{1}{s}\left(\hat{\mathbf{G}}+\hat{\mathbf{G}}^{\prime}\right) \mathbf{E}$, where $\left[\hat{\mathbf{G}}^{\prime} \mathbf{E}\right](\mathbf{r})=-\int_{\text {corner }} d \mathbf{r}^{\prime} \mathbf{G}_{0}\left(\mathbf{r}, \mathbf{r}^{\prime}\right)$ $\mathbf{E}\left(\mathbf{r}^{\prime}\right) k_{0}^{2}$. If $s^{(1)}$ is the first order of correction of the eigenvalue $s^{(0)}$ associated with $d$ degenerated eigenmodes $\left\{\mathbf{E}_{i}, l\right.$ $=1,2 \ldots d\}$, we have

$$
\left|\mathbf{G}_{l, l^{\prime}}^{\prime}-s^{(1)} \delta_{l, l^{\prime}}\right|=0 \text {. }
$$

In the case of the two dipole modes of the square flake, Eq. (5) has two different real roots. In other words, the degenerated level splits.

One interesting phenomenon in Fig. 1(c) is that the amplitude of the mode shift is linked with the local field intensity at the removed corner. The mode which originally has small field intensity at the corner is almost unchanged, while the other mode which presents strong local electric field intensity at the perturbed zone is clearly shifted. This implies that the perturbation induced resonance shift depends on the local field intensity of the resonance mode. Actually, this effect has been observed in closed packed plasmonic dimmer structures and is important for designing ultrasensitive LSPR-based sensors. ${ }^{16-18}$

In addition to mode splitting, breaking the structure symmetry may turn on a dark mode, which appears as new mode in Fig. 1(c). As previously mentioned, whether a mode of a symmetric structure is dark or not depends on whether the eigenmode contains the base of the corresponding unit irrep. When the symmetry of the structure is lowered, the eigenmode might no longer be the base of the symmetry transformations and consequently the integrals in Eq. (4) do not vanish anymore.

The previous discussion is based on the assumption that the external field is constant inside the nanostructure. This assumption however becomes inappropriate when $\mathbf{E}_{\text {ext }}\left(\mathbf{r}_{0}\right)$ in Eq. (3) is zero or small enough to be comparable with the variation of $\mathbf{E}_{\text {ext }}$ in the structure. In this case, the spatial dispersion of $\mathbf{E}_{\text {ext }}$ must be considered and the selection rules are determined by $\left\langle\mathbf{E}_{n} \mid \mathbf{E}_{e x t}\right\rangle$ instead of $\mathbf{E}_{n}$. The full selection rules become: if the $i$ th component of $\mathbf{E}_{n}$, respectively, $\mathbf{E}_{\text {ext }}$,
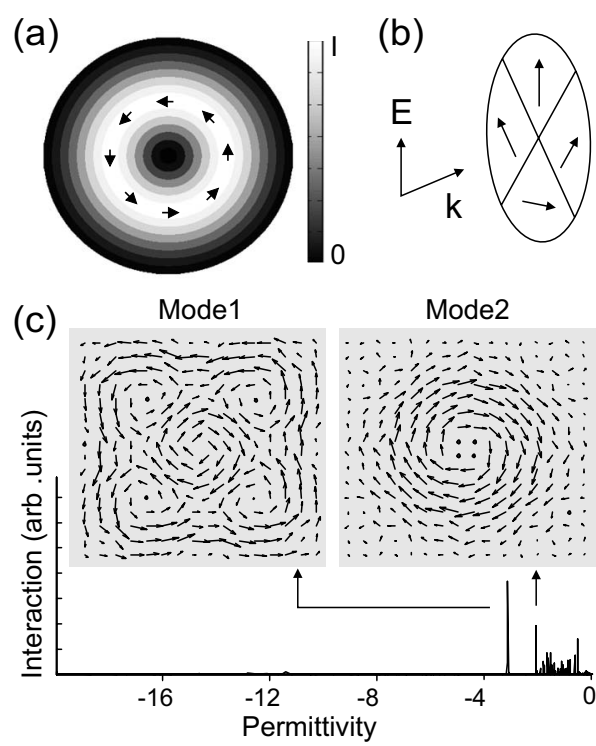

FIG. 2. (a) Field intensity profile of an azimuthally polarized donut shape beam. This type of beam can be generated by using a composited $\lambda / 2$ waveplate (b). (c) Excitation strength as a function of the permittivity of the different eigenmodes of the same square flake as in Fig. 1(a) for the donut shape beam illumination. The insets in (c) show the electric field distributions of the two dominant modes.

transform as the representations $\Gamma_{n, i}$, respectively, $\Gamma_{\text {ext, }}$ $(i=1,2$, or 3$)$, then $\left\langle\mathbf{E}_{n} \mid \mathbf{E}_{\text {ext }}\right\rangle$ vanishes unless there is a $\Gamma_{n, i}$ $\otimes \Gamma_{e x t, i}$ transforming as the unit irrep of the system. Note that when the external field is homogeneous, $\Gamma_{\text {ext }}$ is the unit irrep and the selection rules are solely determined by $\Gamma_{n}$, as was deduced previously.

To illustrate the influence of the external field, we consider an azimuthally polarized donut shape beam, Fig. 2(a). This type of beam can be created by using the composite waveplate depicted in Fig. 2(b). ${ }^{19}$ The field distribution $\mathbf{E}_{e x t}$ inside the square particle carries the irrep $A_{2 g}$ which correspond to the $A_{2}$ mode in Fig. 1(a). Figure 2(c) shows the modes obtained by simulation. The three orthogonal dominant dipole modes in Fig. 1(b) vanish because the direct product of $\Gamma_{n, i}$ and $\Gamma_{e x t, i}$ does not contain any unit irrep component. On the contrary, the dark modes carrying irrep $A_{2 g}$ can be excited by the donut shape beam-see the mode profiles in Fig. 2(c) - because the product $\Gamma_{A_{2 g}} \otimes \Gamma_{A_{2 g}}=\Gamma_{A_{1 g}}$ is the unit irrep.

Another important case in which the approximation of a homogenous external field is invalid is the near-field illumination, which can produce very large field gradients. Here, we consider the field generated by a dipole source as an example. When an oscillating dipole is nearly touching the structure, the gradient of the electric field diverges and becomes strong enough to lighten all the dark modes, as shown in Fig. 3(a). This phenomenon has been utilized to excite the antisymmetric mode in order to create a unidirectional beam with an optical antenna by Kall and his colleague. ${ }^{7}$ This type of excitation of dark-modes only happens when the dipole source is close to the structure. When the dipole-sample distance increases, the gradient of the electric field drops dra- 


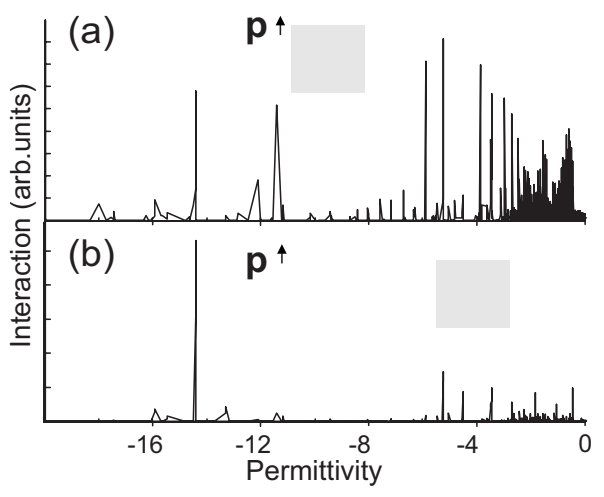

FIG. 3. Excitation spectrum of the nanometer-sized square flake for a dipolar illumination. The spectra are calculated for a dipolesample distance of (a) $1 \mathrm{~nm}$ and (b) $100 \mathrm{~nm}$.

matically and is not large enough to excite the dark modes.

In the above discussion, we have seen that the excitation of LSPRs strongly depends on the spatial distribution of the external electric field which indeed changes strongly from one experiment to the other. Especially in the case of single particle measurements, a tightly focused beam or a near-field scanning optical microscope are often used; the corresponding external field may vary extremely rapidly in space and cause unexpected results. This has however largely been ignored. Actually, Mojarad et al. have pointed out that, even in a case as simple as a spherical nanoparticle, a tightly focused beam and a plane wave generate different results. ${ }^{20}$ In fact, we can even go one step further and selectively excite a dark mode by choosing the proper symmetry of the external fields, as demonstrated in Fig. 2.

To summarize, we have established a general framework to describe how localized surface plasmon modes interact with an external electric field. By implementing the group theory upon the electric vector field $\mathbf{E}$, the selection rules of LSPRs under different illumination conditions have been obtained and further demonstrated for the case of a model square flake. The influence of symmetry breaking on the splitting of degenerated modes and the switching of dark modes has been discussed. These results emphasize the fact that the selection rules for a vector field are different from the case of a scalar field, because a symmetry operator acts on both the position vector and the field vector. All the selection rules are essentially induced by the geometry of the structure. Finally, this work not only points out that measurements of LSPRs may result in very different results with different external fields, but also provides a strategy to selectively excite specific LSPRs of plasmonic structures.

Funding from the Swiss Competence Center for Materials Science and Technology (project Zonop) is gratefully acknowledged.
*Corresponding authors

†weihua.zhang@epfl.ch

*www.nanophotonics.ch

§olivier.martin@epfl.ch

${ }^{1}$ C. F. Bohren and D. R. Huffmann, Absorption and Scattering of Light by Small Particles (Wiley, New York, 1983).

${ }^{2}$ D. P. Fromm, A. Sundaramurthy, P. J. Schuck, G. Kino, and W. E. Moerner, Nano Lett. 4, 957 (2004).

${ }^{3}$ P. Muhlschlegel, H. J. Eisler, O. J. F. Martin, B. Hecht, and D. W. Pohl, Science 308, 1607 (2005).

${ }^{4}$ D. J. Bergman and M. I. Stockman, Phys. Rev. Lett. 90, 027402 (2003).

${ }^{5}$ H. Wang, Y. P. Wu, B. Lassiter, C. L. Nehl, J. H. Hafner, P. Nordlander, and N. J. Halas, Proc. Natl. Acad. Sci. U.S.A. 103, 10856 (2006).

${ }^{6}$ G. Volpe, S. Cherukulappurath, R. J. Parramon, G. MolinaTerriza, and R. Quidant, Nano Lett. 9, 3608 (2009).

${ }^{7}$ T. Pakizeh and M. Kall, Nano Lett. 9, 2343 (2009).

${ }^{8}$ D. J. Bergman and D. Stroud, Solid State Phys. 46, 147 (1992).

${ }^{9}$ M. I. Stockman, S. V. Faleev, and D. J. Bergman, Phys. Rev. Lett. 87, 167401 (2001).
${ }^{10}$ Y. Gu, L. L. Chen, H. X. Zhang, and Q. H. Gong, EPL 83 27004 (2008).

${ }^{11}$ O. J. F. Martin, C. Girard, and A. Dereux, Phys. Rev. Lett. 74 526 (1995).

${ }^{12}$ L. D. Landau and E. M. Lifshitz, Quantum Mechanics (Pergamon Press, Oxford, 1977).

${ }^{13}$ K. Sakoda, Optical Properties of Photonic Crystals (SpringerVerlag, Berlin, 2001).

${ }^{14}$ B. Gallinet, M.-A. Dupertuis, J. Kupec, and B. Witzigmann, J. Opt. Soc. Am. B 27, 1364 (2010).

${ }^{15}$ S. Dalessi and M.-A. Dupertuis, Phys. Rev. B 81, 125106 (2010).

${ }^{16}$ A. Alù and N. Engheta, Nat. Photonics 2, 307 (2008).

${ }^{17}$ P. K. Jain and M. A. El-Sayed, Nano Lett. 8, 4347 (2008).

${ }^{18}$ W. H. Zhang, L. N. Huang, C. Santschi, and O. J. F. Martin, Nano Lett. 10, 1006 (2010).

${ }^{19}$ L. Novotny and B. Hecht, Principles of Nano-Optics (Cambridge University Press, New York, 2006).

${ }^{20}$ N. M. Mojarad, V. Sandoghdar, and M. Agio, J. Opt. Soc. Am. B 25, 651 (2008). 